\begin{tabular}{|c|c|}
\hline Citation & $\begin{array}{l}\text { Yang Zhang, Patrick Reynaert, (2017), } \\
\text { A High-Efficiency Linear Power Amplifier for } 28 \mathrm{GHz} \text { Mobile } \\
\text { communications in } 40 \mathrm{~nm} \text { CMOS } \\
\text { RFIC }\end{array}$ \\
\hline Archived version & $\begin{array}{l}\text { Author manuscript: the content is identical to the content of the published } \\
\text { paper, but without the final typesetting by the publisher }\end{array}$ \\
\hline \multicolumn{2}{|l|}{ Published version } \\
\hline \multicolumn{2}{|l|}{ Journal homepage } \\
\hline \multirow[t]{2}{*}{ Author contact } & Yang.zhang@esat.kuleuven.be \\
\hline & Klik hier als u tekst wilt invoeren. \\
\hline
\end{tabular}

(article begins on next page) 


\title{
A High-Efficiency Linear Power Amplifier for 28GHz Mobile Communications in $40 \mathrm{~nm}$ CMOS
}

\author{
Yang Zhang, Patrick Reynaert \\ KU Leuven ESAT/MICAS, Kasteelpark Arenberg 10, 3001 Heverlee, Belgium \\ \{yang.zhang, patrick. reynaert\}@esat.kuleuven.be
}

\begin{abstract}
This paper presents a high-efficiency, linear power amplifier (PA) for $28 \mathrm{GHz}$ mobile communications in 40nm CMOS technology. The design and layout are optimized for high linearity while maintaining high gain and output power. A capacitance neutralized differential pair with source degeneration inductor for linearity enhancement is discussed. The inductive degeneration technique greatly increases the optimal load impedance, which enables a low loss parallel power combining. The complete PA achieves a measured saturated output power of $18.1 \mathrm{dBm}$ with $41.5 \%$ power-added efficiency (PAE). With 6 Gb/s QAM-64 signals, the proposed $P A$ achieves an average output power of 8.4dBm and 8.8\% PAE, with -25 dBc EVM. All measurements are performed with a fixed bias condition.
\end{abstract}

Index Terms-CMOS, power amplifier, inductive degeneration, linearity, EVM, 5G.

\section{INTRODUCTION}

Several frequency bands in millimeter-wave (mmWave) range have been licenced for future fifth generation (5G) wireless communication systems, enabling $\mathrm{Gb} / \mathrm{s}$ data rate with low latency. To meet the demand of large data traffic within limited spectrum resources, high order modulation scheme like 64 QAM will be adopted, which presents large peak-to-average power ratio (PAPR).

To amplify these modulated signals with high fidelity, PA designs for $5 \mathrm{G}$ applications have to meet the stringent AM-AM and AM-PM linearity requirements. In RF and microwave range, multiple correction techniques have been investigated to improve linearity of the front-ends. However, it is challenging to deploy these techniques into 5G applications, such as phased arrays and MIMO systems, where the baseband bandwidth is relatively large and the function units might be distributed into the systems. Digital pre-distortion (DPD) for each PA can be prohibitively complex for large-scale arrangement. In any case, the nonlinearity property of the PA itself needs to be dealt with in the first place.

Recently, lots of efforts have been put to improve the PA performance for $5 \mathrm{G}$ applications. In the work of [1], high efficiency and linearity are achieved by using subthreshold biasing and inductive degeneration. And as a variable, the size of the transistor is optimized for $-25 \mathrm{dBc}$ EVM. In [2], the linearity is greatly improved by using second harmonic control and deep class-AB biasing. However, the overall performance of the PA, in terms of
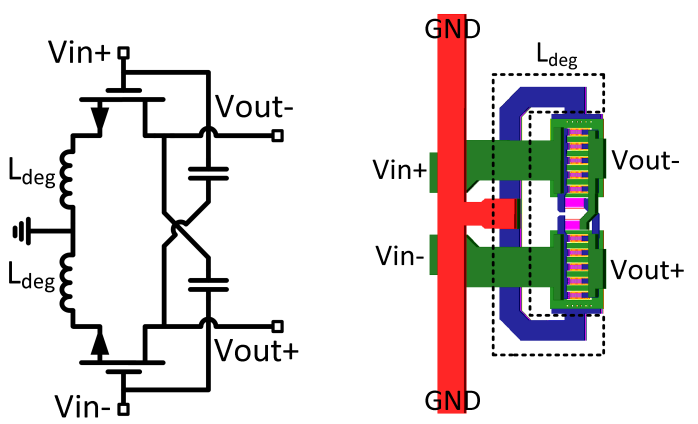

Fig. 1. Schematic of the differential CS pair with source degeneration inductor (left) and its layout (right). The inductor $\left(\mathrm{L}_{\mathrm{deg}}\right)$ is implemented with metal traces from metal-2 to metal-8.

gain, output power, and efficiency can be further improved.

In this paper, we present a high-efficiency, linear power amplifier utilizing source degeneration inductors and lowloss parallel output power combiner. The schematic of the amplifier stage and its layout are shown in Fig. 1. The design procedure targets for minimal AM-AM and AMPM distortion and no biasing tuning or DPD is involved. In section II, the design details are discussed. The circuit implementation is presented in section III. Measurement results are shown in section IV.

\section{Design Details}

Advanced CMOS process with nano-scaled gate is able to provide more gain in millimetre-wave range. On the other hand, the available gain is limited by stability but can be further boosted when capacitance neutralization is utilized to solve the stability issues of the differential common source (CS) pair [3]. Together with inductive degeneration, trade-off can be performed for linearity and efficiency enhancement, while enough gain can still be provided.

\section{A. Source Degeneration Inductor}

As a feedback technique, inductive degeneration has been studied in low noise amplifier and PA designs [1] [3]. When referred back to the gate input impedance, the 


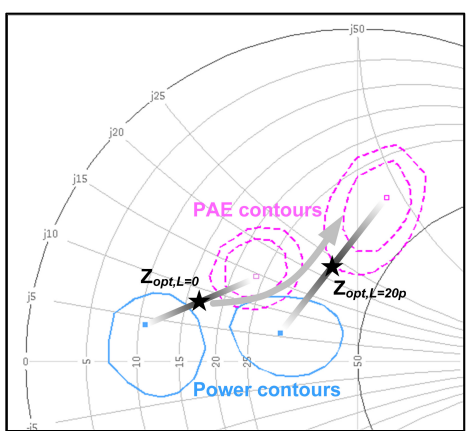

Fig. 2. Grey arrow shows the moving trend of the optimal load impedance as source inductance increases from zero to $20 \mathrm{pH}$.

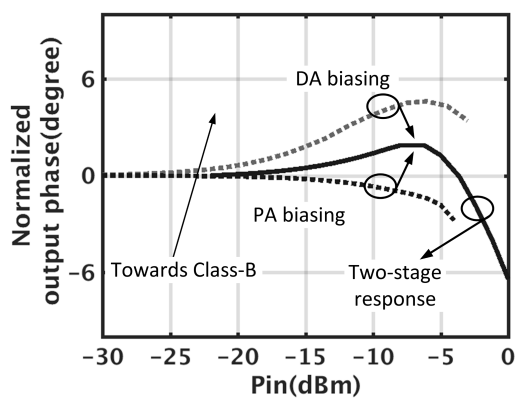

Fig. 3. AM-PM distortion with different biasing conditions for a capacitance neutralized differential pair at $27 \mathrm{GHz}$.

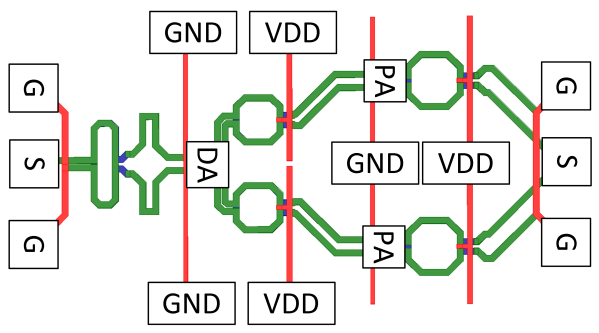

Fig. 4. Simplified layout of the proposed PA. DA and PA unit details are shown in Fig. 1.

source degeneration inductance is transformed into real impedance. Thus the input matching pressure can be released. Compared with resistive and capacitive degeneration, inductive negative feedback exhibits better linearity [3]. The operation $\mathrm{P}_{1 \mathrm{~dB}}$ point is pushed into high input power level, closer to $\mathrm{P}_{\text {sat }}$. Although compared with normal CS pair, the gain is lower, the power back off efficiency and $\mathrm{P}_{1 \mathrm{~dB}}$ are greatly enhanced.

Another key advantage is that the degeneration inductor could greatly increase the optimal shunt load impedance of the last stage, which enables a low loss parallel power combining to further increase the output power. As for the PA designed for higher output power, the loss in the output power combining network plays a dominant role to the overall PA efficiency. The track of the optimal load impedance on the smith chart is shown in Fig. 2. The required shunt load resistance increases by a factor of 2.3 as $20 \mathrm{pH}$ inductive degeneration is applied. After including the extracted local parasitics, an optimal impedance of $66 \Omega$ load resistance is chosen for two $19.2 \mathrm{pH}$ degeneration inductors. A parallel power combining with transformer matching network is used to extract the maximal power. Simulation shows the insertion loss of the entire output network is less than $0.8 \mathrm{~dB}$, at $27 \mathrm{GHz}$, and from $23.5 \mathrm{GHz}$ to $34.5 \mathrm{GHz}$ the loss is better than $0.9 \mathrm{~dB}$.

\section{B. Power Amplifier Linearization}

In order to enhance the efficiency of millimetre-wave amplifiers, the trend is to bias the PAs toward Class-B, which exhibits better energy efficiency than Class-A. However, deep Class-AB and Class-B biasing bring along with large distortion (gain and phase expansion when $\mathrm{P}_{\text {out }}$ is close to $\mathrm{P}_{1 \mathrm{~dB}}$ ), which degrades the error vector magnitude (EVM) and the adjacent channel power ratio (ACPR). The AM-PM distortion against different bias conditions is shown in Fig. 3. It can be seen that there is an optimal biasing point for minimal AM-PM distortion, assuming no other technique is employed. However, this particular optimal bias level is not optimal in the view of efficiency and output power perspectives. For a two stage CS PA design, the proposed PA design utilizes different biasing for the DA stage and PA stage to compensate the overall AM-PM distortion. As mentioned before, the degeneration inductor can extend the linear operation region. In addition, by combining a gain-expansion stage and a gain-compression stage, the AM-AM distortion can be further reduced [2].

\section{CIRCUIT IMPLEMENTATION}

The simplified layout of the linear two-stage PA that utilizes parallel power combiner is shown in Fig. 4, DA and PA units shown in Fig. 1. The matching for inter-stage is performed by a transmission line based power divider together with transformers. At the output the parallel power combiner transfers splits $50 \Omega$ termination into two $58 \Omega$ and then they are matched to the optimal load impedance by transformers. In the EM simulation the dummies of the thick metal layers are simplified and included to estimate their influence and the dummies in Xlayers are ignored.

The differential CS pair is neutralized by two $44 \mathrm{fF}$ capacitors. The transistor unit has a total dimension of $178 \mu \mathrm{m} / 40 \mathrm{~nm}$. The local transistor layout in [4] is adopted to reduce the device parasitics, and M1-M3 layers are used for local ground to reduce the IR drop. The ground planes connect to the degeneration inductors from both sides. To meet the current density requirement M2-M8 metal layers are used. Small slots are put into the X-layers to avoid 


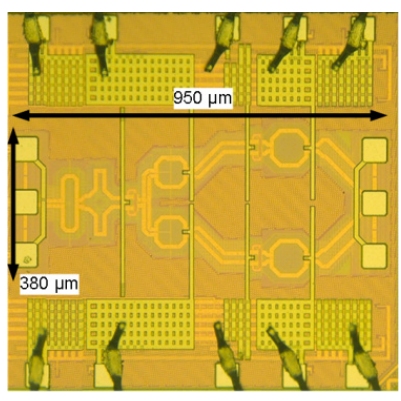

Fig. 5. Die photo of the proposed PA in 40nm CMOS.

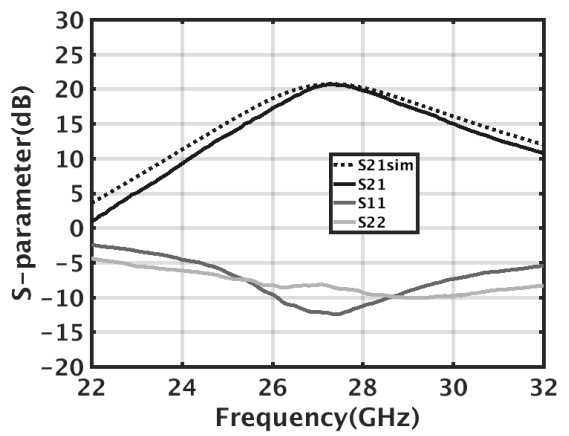

Fig. 6. Measurement results of S-parameters versus frequency.

DRC violation. The inductors from two sides meet at the virtual ground and then connect to aluminium layer. The inductors are placed vertical to the differential input to minimize the unwanted coupling.

\section{MEASUREMENT RESULTS}

The proposed design is fabricated in standard $40 \mathrm{~nm}$ CMOS technology and the die photo is shown in Fig. 5. During the whole measurement, the PA biasing is fixed and $1 \mathrm{~V}$ is used as the supply voltage. The supply and biasing pads are wire-bonded on FR4 substrate. The R\&S VNA and GSG probes with $150 \mu \mathrm{m}$ pitch are used to measure the signal input and output. The measured Sparameter results are shown in Fig. 6. The measurement agrees well with simulation. The gain at $27 \mathrm{GHz}$ is $20.5 \mathrm{~dB}$ with S12 lower than $-50 \mathrm{~dB}$. The dummy fillings in all layers slightly change the equivalent permittivity and generate parasitics which are considered to be reflected on the frequency response. The large signal behaviour is performed at $27 \mathrm{GHz}$, with help of a Keysight PSG and R\&S power meter. The measured results are shown in Fig. 7 and the power consumption versus output power is given in Fig. 8. The proposed PA achieves a $16.8 \mathrm{dBm}$ output power at $\mathrm{P}_{1 \mathrm{~dB}}$ point with $37.6 \% \mathrm{PAE}$ and the maximum output power is $18.1 \mathrm{dBm}$ with $41.5 \% \mathrm{PAE}$. The maximum AM-PM variation is less than 2-degrees till $\mathrm{P}_{1 \mathrm{~dB}}$ point and gain expansion in the linear region is less than $0.35 \mathrm{~dB}$. The results indicate that the PA linearity is greatly

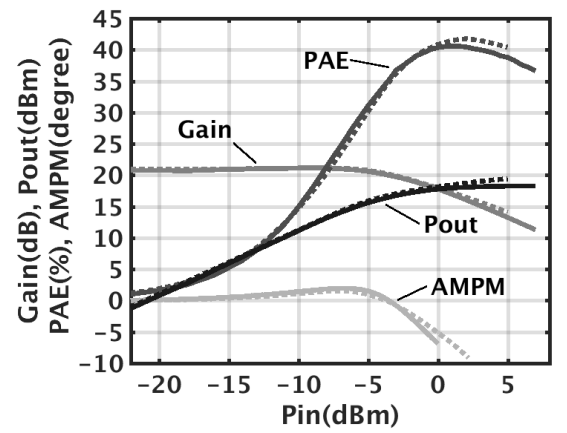

Fig. 7. Measured gain, output power, PAE and AM-PM distortion versus input power at $27 \mathrm{GHz}$. (dotted line: simulation)

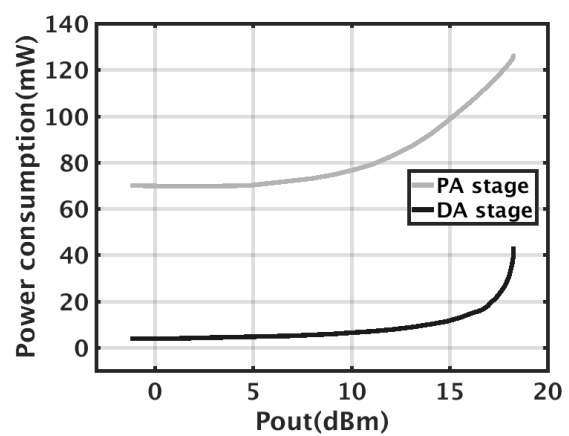

Fig. 8. Measured DC power consumption versus output power at $27 \mathrm{GHz}$.

improved while the high efficiency and output power performance can still be maintained.

To further characterize the PA, modulated signal measurements are performed. QAM-64 signals with a PAPR of $8.3 \mathrm{~dB}$ are generated by a Keysight M8195A AWG and a raised-cosine shaped filter with a rolloff factor of 0.35 is used. The signals are up-converted and apply to the input of the PA by probes. The output port connects to a Keysight oscilloscope, which has built-in VSA software to analyse the amplified modulated signals. All the passive losses in the input and output path are carefully taken care. The setup is calibrated without the DUT to eliminate the image effect and LO feed-through. Fig. 9 shows the measured constellation and spectrum with a span of $2 \mathrm{GHz}$ and $3 \mathrm{GHz}$ and EVM performance. The EVM is normalized to the reference RMS power. The measured average output power for $4 \mathrm{~Gb} / \mathrm{s}$ data rate is $8.8 \mathrm{dBm}$ with $9.6 \%$ average $\mathrm{PAE}$, and $8.4 \mathrm{dBm}$ with $8.8 \%$ average PAE for data rate of $6 \mathrm{~Gb} / \mathrm{s}$. It can be seen that although the signal is slightly compressed, $-25 \mathrm{dBc} E V M$ can still be maintained. Table I summarizes the measured results and gives a comparison with state-of-the-art PA designs. This PA achieves the highest Figure-of-Merit (FoM) at $28 \mathrm{GHz}$ frequency range and could deliver the highest data rate of $6 \mathrm{~Gb} / \mathrm{s}$ with $-25 \mathrm{dBc}$ EVM. 

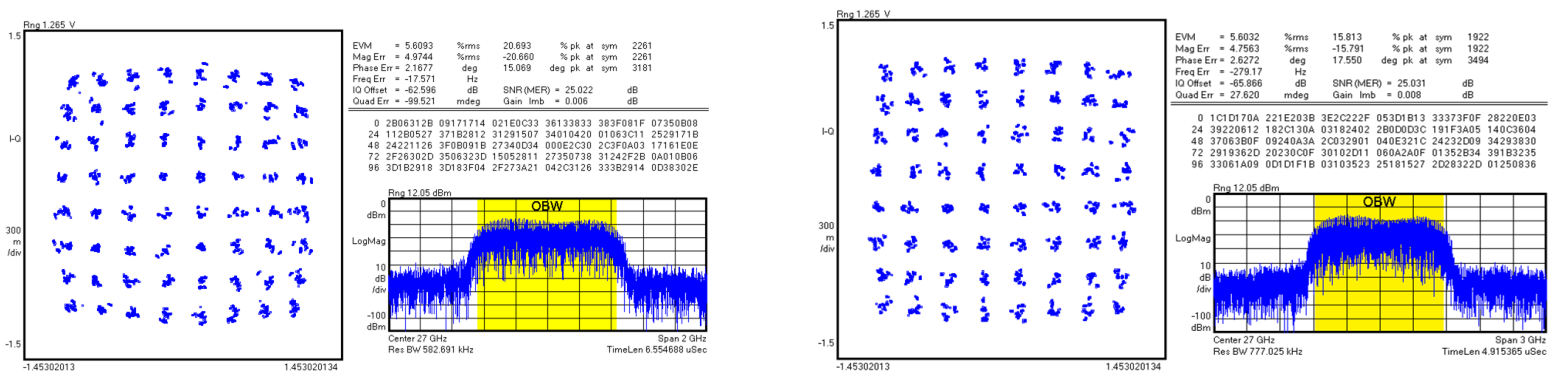

Fig. 9. Measured constellation, spectrum (2GHz and $3 \mathrm{GHz}$ span) and EVM of QAM-64 modulation, 4Gb/s (a) and 6Gb/s (b).

TABLE I

COMPARISON OF STATE-OF-THE-ART PAS

\begin{tabular}{|c|c|c|c|c|c|c|c|c|c|}
\hline Ref. & \multicolumn{3}{|c|}{ This work $^{\#}$} & $\mathrm{JSSC}^{\prime} 16^{\#}[1]$ & \multicolumn{2}{|c|}{ TMTT'16[2] } & ISSCC'14[5] & JSSC'13[4] & SiRF'14[6] \\
\hline Frequency $[\mathrm{GHz}]$ & \multicolumn{3}{|c|}{27} & 30 & \multicolumn{2}{|c|}{28.5} & 63 & 61 & 28 \\
\hline Technology & \multicolumn{3}{|c|}{ 40nm CMOS } & $28 \mathrm{~nm} \mathrm{CMOS}$ & \multicolumn{2}{|c|}{ 28nm CMOS } & $40 \mathrm{~nm} \mathrm{CMOS}$ & $40 \mathrm{~nm} \mathrm{CMOS}$ & $120 \mathrm{~nm} \mathrm{SiGe}$ \\
\hline Vdd [V] & \multicolumn{3}{|c|}{1} & 1 & 1.1 & 2.2 & 1.8 & 1 & 3.6 \\
\hline Gain $[\mathrm{dB}]$ & \multicolumn{3}{|c|}{20.5} & 15.7 & 10.1 & 13.6 & 22.4 & 17 & 15.3 \\
\hline $\mathrm{P}_{1 \mathrm{~dB}}[\mathrm{dBm}]$ & \multicolumn{3}{|c|}{16.8} & 13.2 & 14 & 18.6 & 13.9 & 13.8 & 15.5 \\
\hline $\mathrm{PAE}_{1 \mathrm{~dB}}[\%]$ & \multicolumn{3}{|c|}{37.6} & 34.3 & 35.2 & 41.4 & 18.9 & 21.6 & 31.5 \\
\hline $\mathrm{P}_{\text {sat }}[\mathrm{dBm}]$ & \multicolumn{3}{|c|}{18.1} & 14 & 14.8 & 19.8 & 16.4 & 17 & 18.6 \\
\hline $\mathrm{PAE}_{\mathrm{MAX}}[\%]$ & \multicolumn{3}{|c|}{41.5} & 35.5 & 36.5 & 43.3 & 23 & 30.3 & 35.3 \\
\hline $\mathrm{FoM}^{*}$ & \multirow{2}{*}{\multicolumn{3}{|c|}{$\begin{array}{c}83.4 \\
<2\end{array}$}} & 74.7 & 69.4 & 78.7 & 88.4 & 84.4 & 78.3 \\
\hline AM-PM [deg] & & & & $<6^{\# \#}$ & \multicolumn{2}{|c|}{-} & 0.2 & 3 & - \\
\hline Modulated signal & \multicolumn{3}{|c|}{ QAM-64 } & $\begin{array}{c}\text { QAM-64 } \\
\text { OFDM } \\
\end{array}$ & \multicolumn{2}{|c|}{$\begin{array}{l}\text { QAM-64 } \\
\text { (WLAN) }\end{array}$} & QAM-64 & - & - \\
\hline Data rate $[\mathrm{Gb} / \mathrm{s}]$ & 1.5 & 4 & 6 & 1.5 & \multicolumn{2}{|c|}{$<0.48$} & 3 & - & - \\
\hline $\operatorname{EVM}^{* *}[\mathrm{dBc}]$ & \multicolumn{3}{|c|}{-25} & -25 & -27.4 & -27.5 & -25 & - & - \\
\hline $\mathrm{P}_{\text {out }} @$ EVM $[\mathrm{dBm}]$ & 9.65 & 8.8 & 8.4 & 4.2 & 6.77 & 10.97 & 7 & - & - \\
\hline PAE @ EVM [\%] & 11.8 & 9.6 & 8.8 & 9 & 16.45 & 17.3 & 5 & - & - \\
\hline
\end{tabular}

${ }^{*} \mathrm{FoM}=\mathrm{P}_{\text {sat }}[\mathrm{dBm}]+\mathrm{Gain}[\mathrm{dB}]+20 \log ($ freq[GHz] $)+10 \log \left(\mathrm{PAE}_{\mathrm{MAX}}[\%]\right) .{ }^{* * N o r m a l i z e d ~ t o ~ t h e ~ r e f e r e n c e ~ R M S ~ p o w e r . ~}(\mathrm{EVM} \approx-\mathrm{SNR}(\mathrm{MER}))$

\# Fixed biasing condition for all measurement.

\section{CONCLUSION}

A high-efficiency, linear CMOS power amplifier is presented for $28 \mathrm{GHz}$ mobile applications. Source inductive degeneration is adopted to improve the linearity and efficiency. The proposed design is optimized for minimal distortion while high efficiency and output power are still maintained. A low loss parallel power combiner is applied to further increase the output power. Measurement results show that the proposed PA achieves state-of-the-art performance in terms of gain, efficiency and output power. Thanks to the linearity enhancement design, the proposed PA managed to deliver QAM-64 signals with the highest data rate of $6 \mathrm{~Gb} / \mathrm{s}$ and achieves an average output power of $8.4 \mathrm{dBm}$ and $8.8 \%$ PAE, without any biasing tuning.

\section{ACKNOWLEDGMENT}

This work is supported by the European Commission in the framework of the H2020-ICT-2014-2 project Flex5Gware (Grant agreement no. 671563).

\section{REFERENCES}

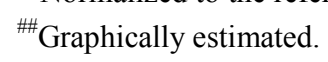

[1] S. Shakib et al., "A Highly Efficient and Linear Power Amplifier for 28-GHz 5G Phased Array Radios in 28-nm CMOS," IEEE J. Solid-State Circuits, vol. 51, no. 12, pp. 3020-3036, Dec. 2016.

[2] B. Park et al., "Highly Linear mm-Wave CMOS Power Amplifier," IEEE Trans. Microw. Theory Techn., vol. 64, no. 12, pp. 4535-4544, Dec. 2016.

[3] K. L. Fong and R. G. Meyer, "High-frequency Nonlinearity Analysis of Common-emitter and Differential-pair Transconductance stages," IEEE J. SolidState Circuits, vol. 33, no. 4, pp. 548-555, Apr. 1998.

[4] D. Zhao and P. Reynaert, "A 60-GHz Dual-Mode Class AB Power Amplifier in 40-nm CMOS," IEEE J. SolidState Circuits," vol. 48, no. 10, pp. 2323-2337, Aug. 2013.

[5] S. Kulkarni and P. Reynaert, "A Push-pull mm-Wave Power Amplifier with $<0.8^{\circ}$ AM-PM Distortion in $40 \mathrm{~nm}$ CMOS," IEEE Int. Solid-State Circuits Conf. (ISSCC) Dig. Tech. Papers, pp. 252-253, Feb. 2014.

[6] A. Sarkar and B. Floyd, "A 28-GHz class-J Power Amplifier with 18-dBm Output Power and 35\% Peak PAE in 120-nm SiGe BiCMOS," in Proc. IEEE $14^{\text {th }}$ Topical Meeting Silicon Monolithic Integr. Circuits RF Syst. (SiRF), pp. 71-73, Jan. 2014. 